Research Paper

\title{
RNA Interference-Mediated Aurora Kinase A Gene Silencing Inhibits Human Glioma Cells Proliferation and Tumor Growth in Mice
}

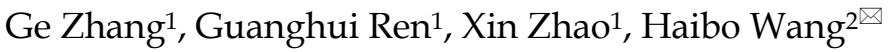 \\ 1. Department of Neurology, Luoyang Dong Fang Hospital, The Third Affiliated Hospital of Henan University of Science \& Technology, Luoyang, Henan \\ 471003, PR China. \\ 2. Institute of Cardiovascular Disease, Ruijin Hospital, Shanghai Jiao Tong University School of Medicine, Shanghai 200025, PR China.
}

$\triangle$ Corresponding author: Haibo Wang. Street address: No. 197 Ruijin 2nd Road, Huangpu District, Shanghai, China. Postal code: 200025; Email Address: wanghaibo@sjtu.edu.cn; Phone: 86-021-64370045

(c) The author(s). This is an open access article distributed under the terms of the Creative Commons Attribution License (https://creativecommons.org/licenses/by/4.0/). See http:/ /ivyspring.com/terms for full terms and conditions.

Received: 2020.11.11; Accepted: 2021.03.04; Published: 2021.03.19

\begin{abstract}
Objective: This study aims to explore the roles of Aurora Kinase A (Aurora A) in human glioma progression and relevant molecular mechanisms involved.

Methods: RNA interference (RNAi) technology was performed to silence the Aurora $A$ gene in human glioma cell line U251 and U87. Western blot and real-time PCR were used to determine the protein and mRNA expression levels of Aurora A. Flow cytometry was performed to analyze the cell cycle distribution and MTT was used to examine the cell viability. Annexin V/FITC double staining and Hoechst 33258 staining were carried out to examine cell apoptosis. Xenograft tumor model was established to examine the effect of Aurora A siRNA on tumor growth in vivo.

Results: RNAi-mediated Aurora A gene silencing with specific short interfering RNA (siRNA) significantly decreased Aurora A protein and mRNA expression levels in human glioma cell line U251 and U87. Aurora A knockdown in glioma cells with siRNA strongly inhibited cell proliferation, along with the accumulation of cells in the G1, G2/M phase and decrease in S phase. Furthermore, the enhancement of cell apoptosis in vitro and the suppression of xenograft tumor growth in vivo were also observed after Aurora A silencing in U251 cell. In addition, Aurora A knockdown resulted in decreased expression of anti-apoptotic protein Bcl-2 and cell cycle protein Cyclin D1, while increased expression of pro-apoptotic factor caspase-3.

Conclusion: Aurora $\mathrm{A}$ can be used as a candidate targeting gene and inhibition of Aurora $\mathrm{A}$ is a potentially promising therapy for glioblastoma.
\end{abstract}

Key words: RNA interference; Aurora Kinase A; Glioma; Proliferation; Apoptosis; Tumor growth.

\section{Introduction}

Glioblastoma (GBM) is the most aggressive and widespread brain tumor in adults. Patients that are diagnosed with GBM exhibit a median survival of merely 12 months, even with aggressive therapies such as chemotherapy, radiation and operative resection [1, 2]. Furthermore, these high-grade gliomas relapse after operative resection with standard chemo- and radiation treatments. Several immunotherapies were applied for glioma cancer's therapy, including administrating antiglioma, growth factor receptor-specific antibodies and immune adjuvants, cytokine transduced glioma cell vaccines and in vitro stimulated tumor that infiltrated lymphocytes (TIL) and lymphokine-activated killer cells (LAK) [3]. A few patients, however, were cured by these immunotherapeutic modalities occasionally. Despite recent progresses in both treatment strategies and diagnostic patterns, glioma continues to be one of the deadliest human cancers [4]. 
Three members are contained by serine/ threonine kinases' Aurora family in mammalian cells, designated Auroras A, B and C, which have in common sequence homology's highest degree in their catalytic domains [5]. Their cellular localization and expression pattern, however, are different markedly. Aurora A kinase is primarily expressed in the cell cycle's G2/M phase and regulates mitotic cell division in normal cells [6,7], which is required for cell division and centrosome maturation [8]. Aurora A is expressed in numerous different human normal tissues, which is, however, aberrantly overexpressed and has been involved in tumorigenesis recently, and correlated with poor prognosis in varieties of cancer patients [9, 10]. Aurora Kinase A, a centrosomeassociated serine/threonine protein kinase, is amplified and overexpressed in several types of human tumors, including glioblastoma. Previous study has demonstrated that Aurora A gene involved in mitotic processes, was associated with poor prognosis in glioblastoma. However, the roles of Aurora A in human glioma progression and relevant molecular mechanisms mediating these effects remain largely unknown.

Aurora A overexpression has been demonstrated in various of human cancers at multiple kinds of pathological stages, including hepatocellular carcinoma (HCC) [11], esophageal squamous cell carcinoma (ESCC) [12], gastrointestinal adenocarcinomas [13], ovarian cancer [9], head and neck squamous cell carcinoma (HNSCC) [10], and glioma $[14,15]$, etc., which has been associated with a poor prognosis of cancer patients. Furthermore, Aurora-B has also been found overexpressed in human gliomas [16]. Aurora kinases' inhibition, especially Aurora A, therefore, is very urgent for treatment's implementation for human glioma. Several small molecule inhibitors have been recently developed revealing anti-proliferative effects on tumor cells in preclinical models. For example, it has been demonstrated that MLN8054 is a selective small-molecule Aurora A kinase inhibitor that has entered Phase I clinical trials for advanced solid tumors [17]. Besides, some highly effective Aurora and pan-Aurora kinase inhibitors (AKI), which were capable to inhibit all aurora kinases, including PHA-739358, has entered phase 2 clinical trials for patients with advanced stages of prostate cancer and hematological malignancies [18], and SNS-314 [19], VX-680 [20] and AZD1152 [21] show efficient anti-tumor activity. Therefore, the small molecules with an Aurora A inhibitory function may make it possible to reduce or inhibit the oncogenic activity of Aurora A [22]. Previous studies have demonstrated that silencing of the gene that encodes Aurora A in tumor cells causes their growth inhibition and enhances the cytotoxicity of anticancer agents [23]. These data powerfully suggest that Aurora A is one of the potential targets and fundamental cancer-related genes for cancer therapy [22, 23].

Aurora A gene expression was the only predictive factor. However, the biological functions of Aurora A in human glioma cells have yet to be well understood. In this study, we examined the effect of Aurora A silencing by RNAi on human glioma cell growth and apoptosis and relevant molecular mechanism involved.

\section{Materials and methods}

\section{Cell culture}

Human glioma cell line U251 and U87 were purchased from American Type Culture Collection (Manassas, VA, USA) and cultured in DMEM (Gibico, Grand Island, NY, USA) supplemented with $10 \%$ FBS (Gibico, Grand Island, NY, USA), penicillin (100 $\mathrm{U} / \mathrm{ml})$ and streptomycin $(0.1 \mathrm{mg} / \mathrm{ml})$. Cells were incubated at $37^{\circ} \mathrm{C}$ with humidified air containing $5 \%$ $\mathrm{CO}_{2}$.

\section{Short interference RNA transfection}

The siRNA oligonucleotide sequence for Aurora A (NCBI: NM_003600) used was supplied by Dharmacon Research Inc. The sequence of siRNA: 5'-GCCGGUUCAGAAUCAGAAG-3'. The sequence of control siRNA: 5'-UUCUCCGAACGUGUCA CGU-3'. siRNA transfection was performed with Lipofectamine 2000 (Invitrogen, NY, USA) according to the manufacturer's instructions. In brief, $24 \mathrm{~h}$ before transfection, U251 and U87 cells were trypsinized and transplated on a 6-well plate at $1^{*} 10^{5}$ cells/well in $2 \mathrm{ml}$ of culture medium supplemented with DMEM and $10 \%$ serum without antibiotics for $24 \mathrm{~h}$. Thereafter, 20 $\mathrm{nM}$ Aurora A siRNA or control siRNA diluted in $50 \mu \mathrm{l}$ of Opti-MEM and $5 \mu 1$ Lipofectamine 2000 diluted in $50 \mu \mathrm{l}$ of Opti-MEM were preincubated for $5 \mathrm{~min}$. The two mixtures were incubated for $25 \mathrm{~min}$ at room temperature for complex formation. $150 \mu \mathrm{l}$ of Opti-MEM was added, then the entire mixture was added to each well. The cells were harvested $48 \mathrm{~h}$ after transfection.

\section{Real-time quantitative PCR}

Total RNA was initially extracted from cultured glioma cells using TRIzol (Invitrogen) and treated with RNase-free DNase I. Standard reverse transcription reaction was carried out using a Promega M-MLV cDNA synthesis kit following the manufacturer's instructions. The real-time reverse transcription polymerase chain reaction was performed using the SYBR Green One-Step qRT-PCR 
kit (Invitrogen) according to the kit's procedure manual. GAPDH was used as an internal control. The sequences of the primers were as follows: GAPDH forward, 5'-TGACTTCAACAGCGACACCCA-3'; GAPDH reverse, 5'-CACCCTGTTGCTGTAGCC AAA-3'; Aurora A forward, 5'-AATGCCCTGTC TTACTGTCATTC-3'; Aurora A reverse, 5'-TCCAGA GATCCACCTTCTCATC-3'. The relative gene expression levels were calculated using the 2- $\Delta \Delta \mathrm{CT}$ algorithm. Each assay was performed in triplicate and the experiment was repeated at least three times.

\section{Western blot}

Glioma cells in the dishes were lysed directly with lysis buffer $(50 \mathrm{mM}$ Tris- $\mathrm{HCl}, 150 \mathrm{mM} \mathrm{NaCl}$, $0.02 \%$ NaN3, 1\% Triton X-100, $1 \mathrm{mM}$ PMSF and 1 $\mu \mathrm{g} / \mathrm{ml}$ aprotinin, $1 \mu \mathrm{g} / \mathrm{ml}$ leupeptin). The protein concentration was assayed by BCA Protein Assay (Pierce, Rockford, IL). Cell lysates containing equal amounts of protein were separated on $10 \%$ sodium dodecyl sulfate-polyacrylamide gel electrophoresis (SDS-PAGE) and electrotransferred onto nitrocellulose (NC) membranes (Millipore Billerica, MA). After overnight blocking with $5 \%$ non-fat dry milk in Tris-buffered saline (TBS) at $4^{\circ} \mathrm{C}$, then the membranes were incubated with the following primary antibodies: anti-Aurora A, anti-Aurora B, anti-Aurora C (Santa Cruz, CA, USA), anti-Bcl-2, anti-Cyclin D1 and anti-Caspase-3 were purchased from Cell Signaling (Cell Signaling, Danvers, MA) and anti- $\beta$-actin (Sigma) at $4^{\circ} \mathrm{C}$ overnight and horseradish peroxidase HRP-conjugated secondary antibodies (Jackson ImmunoResearch, PA) for $2 \mathrm{~h}$ at room temperature. The protein was visualized with ECL detection reagents (BioVision, CA, USA) and then was examined through scanning densitometry using Tanon Image System. Anti- $\beta$-actin antibody was used loading control. The experiment was repeated at least three times.

\section{MTT assay}

Cell proliferation was examined using MTT as previously described [24]. Briefly, U251 and U87 cells were seeded in quadruplicates on 96-well plates at a density of $1 \times 10^{5}$ cells per well in $1 \mathrm{ml}$ medium, then incubated in a $37^{\circ} \mathrm{C}$ humidified incubator for attachment. Cells were transfected with or without Aurora A siRNA / control siRNA after 24, 48 and $72 \mathrm{~h}$ at $37{ }^{\circ} \mathrm{C}$ in a $5 \% \quad \mathrm{CO}_{2}$ and $95 \%$ air-humidified environment, then $20 \mu \mathrm{l}$ of MTT $(5 \mathrm{mg} / \mathrm{ml}$, Sigma, USA) was added to each well, and cells were incubated for another $4 \mathrm{~h}$, then $250 \mu \mathrm{l}$ of dimethyl sulfoxide (DMSO, Sigma, USA) was added and incubated for $30 \mathrm{~min}$ at room temperature for color development. The absorbance values were determined at 1, 2, 3 day in each group respectively. The optical density was determined on a microplate reader at $492 \mathrm{~nm}$ filter (Tecan, Groedig, Austria). Each assay was performed in triplicate and the experiment was repeated at least three times. The relative percentage of cell survival was determined as follows: mean absorbance from Aurora A siRNA or control siRNA group/mean absorbance from parental group $\times 100 \%$.

\section{Cell cycle analysis}

At 3 days after transfection, U251 and U87 cells from parental, Aurora A siRNA or control siRNA treatment group, respectively were collected by centrifugation, washed thrice with cold PBS, and fixed with $70 \%$ ethanol at $4^{\circ} \mathrm{C}$ overnight. The fixed cells were resuspended in PBS containing $10 \mu \mathrm{g} / \mathrm{mL}$ propidium iodide (PI, Sigma, USA) and $100 \mu \mathrm{g} / \mathrm{mL}$ RNase A, then incubated at $4^{\circ} \mathrm{C}$ for at least $30 \mathrm{~min}$ avoiding light to eliminate the endogenous RNA. Cell cycle distribution was determined using flow cytometry (Becton-Dickinson FACScan). Each assay was performed in triplicate and the experiment was repeated at least three times.

\section{Apoptosis assay}

Apoptosis was detected with Annexin V-FITC kit (BD Bioscience, NY, USA) according to the manufacturer's instructions. In brief, at 3 day after transfection, U251 cells from parental, Aurora A siRNA or control siRNA group were trypsinized, collected and washed with cold PBS and centrifuged. Cell pellets were resuspended in cold binding buffer. Annexin V-FITC $(1.25 \mu \mathrm{l} / 0.5 \mathrm{ml})$ and propidium iodide (PI) solution $(10 \mu \mathrm{l} / 0.5 \mathrm{ml})$ were then added. The tubes were incubated for $15 \mathrm{~min}$ in the dark on ice before analysis by flow cytometry (Becton-Dickinson FACScan). Apoptosis was also determined through the fragmented nuclear staining. Cells from various treatment groups were washed with PBS and fixed with ice-cold methanol for $30 \mathrm{~s}$. Cells were stained with Hoechst 33258 (Invitrogen, Grand Island, NY, USA) for $30 \mathrm{~min}$ in the dark before morphological analysis under the fluorescence microscope (Olympus). Each assay was performed in triplicate and the experiment was repeated at least three times.

\section{Liposomal siRNA preparation}

siRNA for in vivo delivery was incorporated into a neutral liposome (1,2-dioleoyl-sn-glycero-3phosphatidylcholine; DOPC). siRNA and DOPC were mixed in the presence of excess t-butanol at a ratio of $1: 10(\mathrm{w} / \mathrm{w})$ as described previously $[25,26]$. Tween 20 was added to the mixture, which was then vortexed, frozen in an acetone/dry ice bath, and lyophilized. Before in vivo administration, this preparation was 
hydrated with normal $(0.9 \%)$ saline at a concentration of $15 \mu \mathrm{g} / \mathrm{mL}$ to achieve the desired dose in 150 to 200 $\mu \mathrm{L}$ per injection.

\section{In vivo xenograft tumor model}

The female nude mice (4 - 6 weeks old, Animal Center of the Chinese Science Academy) were housed in specific pathogen-free conditions. $5 \times 10^{6} \mathrm{U} 251$ cells in $0.1 \mathrm{ml}$ of PBS solution were injected subcutaneously into the right flank of each nude mice. After 7 days, when the tumor volume was about $100 \mathrm{~mm}^{3}$, the mice were randomly subjected to three groups $(n=6)$ for daily intra-tumor injection of liposomal/Aurora A siRNA $(5 \mu \mathrm{g} / 100 \mu \mathrm{l})$, liposomal/control siRNA (5 $\mu \mathrm{g} / 100 \mu \mathrm{l})$ and liposomal/PBS (100 $\mu \mathrm{l}$ as blank) for 21 days. The perpendicular diameters were measured with a caliper every 3 to 4 days, and tumor volume was calculated according to the formula: $(\mathrm{V})=\mathrm{a} \times \mathrm{b}^{2} \mathrm{x}$ 0.5 . After 3 weeks, the mice were sacrificed, and their tumor tissues were harvested. All animal experiments were approved by the Ethics Committee for Animal Use of our hospital.

\section{Statistical analysis}

All results were presented as mean \pm standard deviation (SD). Differences among experimental

(A)

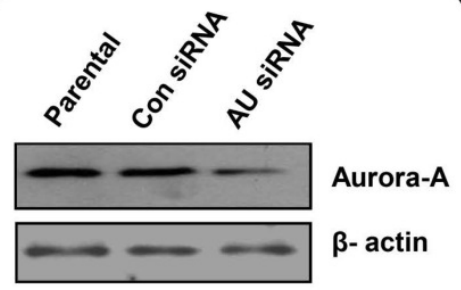

(C)

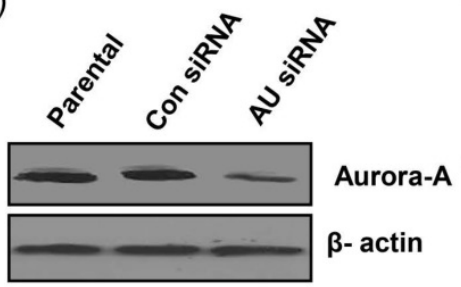

(E)

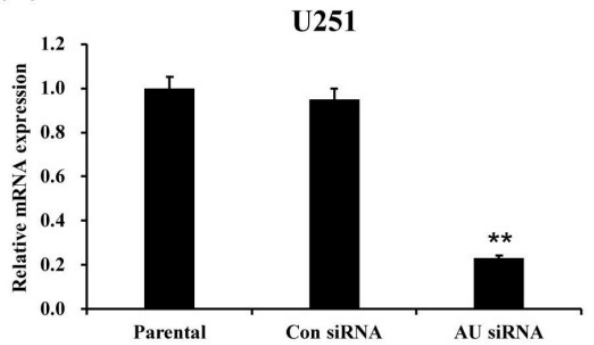

(B)

(D) groups were analyzed for statistical significance by one-way analysis of variance (ANOVA) followed by Bonferroni post hoc tests and the nonparametric tests (Tukey test), Value of $P<0.05$ was considered as significant.

\section{Results}

\section{Specific knockdown of Aurora A in glioma cell line}

Western blot and real-time qPCR analyses were performed after 48 hours transfection. RNAimediated gene silencing significantly reduced the protein and mRNA expression of Aurora A by 80\% and $\sim 75 \%$ as compared to the control siRNA treatment in the U251 (Fig. 1A, B, E) and U87 (Fig. 1C, D, F) cell lines, respectively. In view of the homology of the three Aurora kinases, Aurora A, Aurora B and Aurora $C$, we documented the specificity of the Aurora A silencing, the results showing that the expression of Aurora B and Aurora C are not affected by Aurora A siRNA (Supplemental Fig. 1). These results indicated that Aurora A expression was significantly inhibited by siRNA, neither Aurora B nor Aurora C.
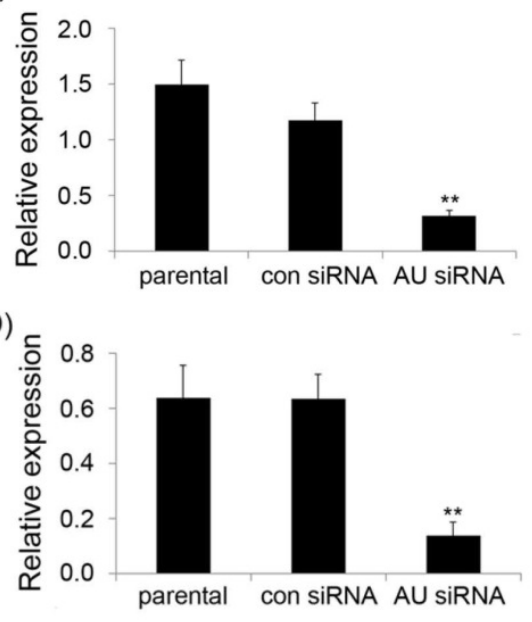

$(\mathrm{F})$

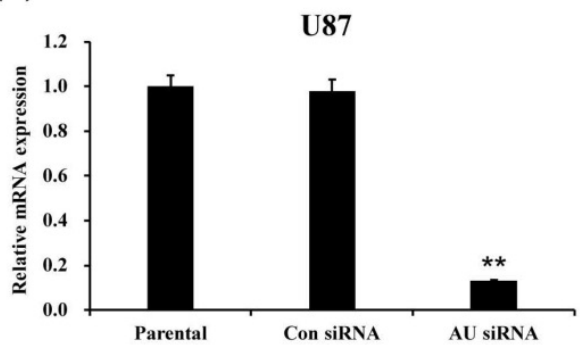

Figure 1. Aurora A silencing with siRNA inhibited its expression in glioma cells. Aurora A protein expression was examined by Western Blot in U251 cells (A) and U87 cells (C). Aurora A protein quantification in U251 cells (B) and U87 cells (D) were also presented, respectively by densitometric analysis of band area. $\beta$-actin served as an internal control. Aurora A mRNA expression was examined by real-time qPCR in U251 cells (E) and U87 cells (F). The expression levels of mRNAs were normalized to that of GAPDH mRNA. The experiments were performed three times with consistent and repeatable results. Each bar represents the mean $\pm S D$, and $* * P<0.01$, AU indicates $A$ urora $A$, con siRNA indicates control siRNA. 
(A)

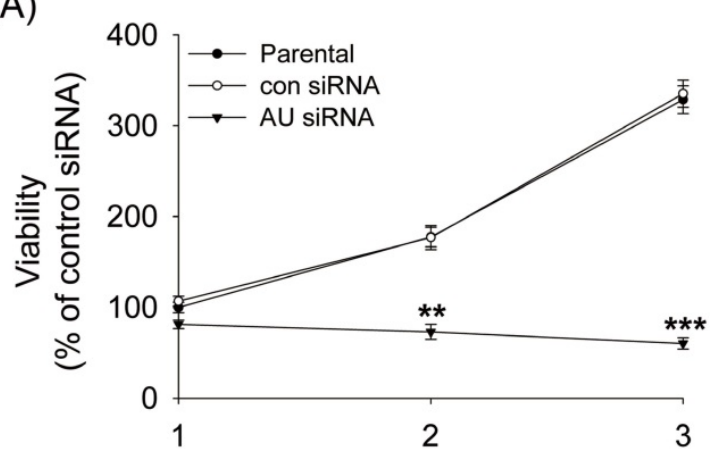

(B)

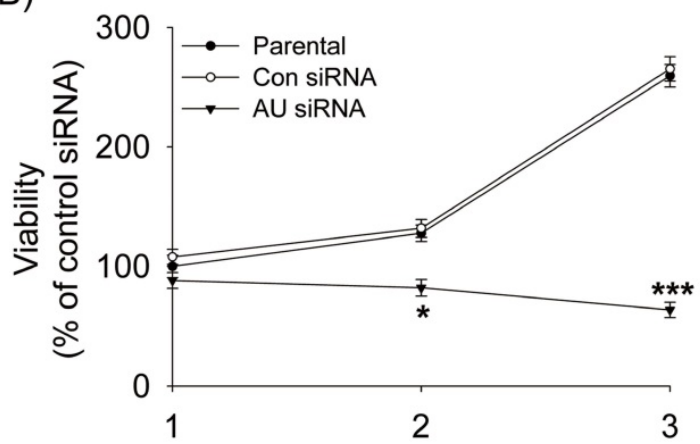

Figure 2. Aurora A silencing with siRNA inhibited cell proliferation. U251 (A) and U87 cells (B) were transiently transfected with Aurora A siRNA, control siRNA or no-treated (parental) for 24,48 , and 72 hours respectively, MTT assay was performed to assess cell proliferation. The experiment data was expressed as mean \pm SD from triplicate and repeated three times independently. $* \mathrm{p}<0.05$, ** $\mathrm{p}<0.01$, **** $\mathrm{p}<$ $0.001, \mathrm{AU}$ indicates Aurora $\mathrm{A}$, con siRNA indicates control siRNA.
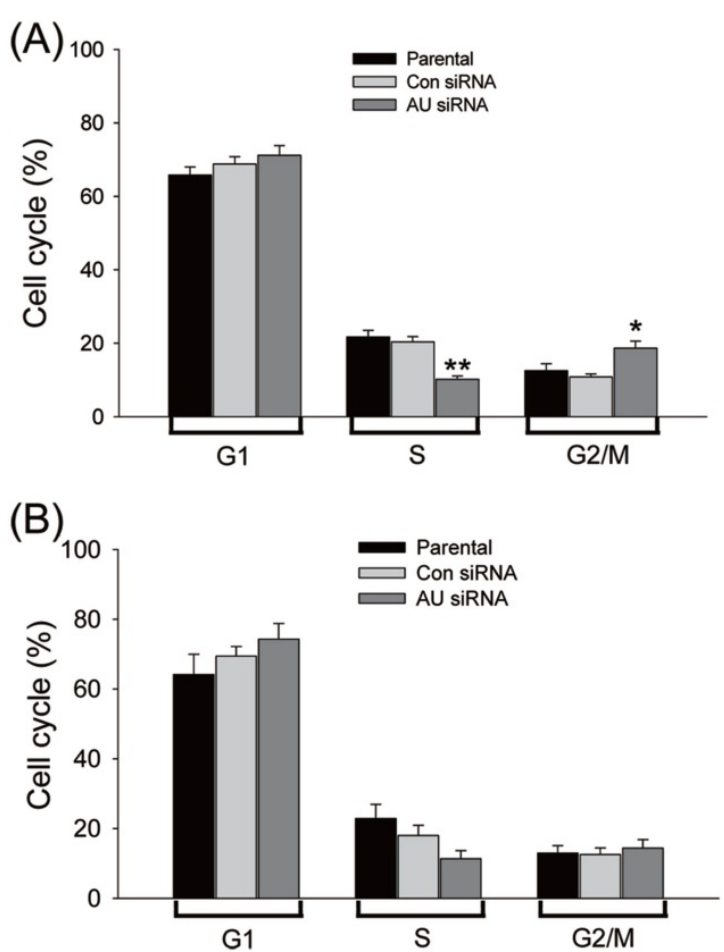

Figure 3. Effects of Aurora $A$ silencing with siRNA disrupted cell cycle progression. Glioma U251 (A) and U87 cells (B) were collected 72 hours after Aurora A siRNA, control siRNA transfection or non-treatment (parental), respectively and subsequently assayed for their DNA content by flow cytometry. The experiments were repeated at least three times independently and the data were expressed as mean \pm SD from triplicate. ${ }^{*} \mathrm{p}<0.05,{ }^{* *} \mathrm{p}<0.01$, AU indicates Aurora $\mathrm{A}$, con siRNA indicates control siRNA.
Effects of Aurora A knockdown on glioma cells proliferation and cell cycle distribution

To further investigate the roles of Aurora A in glioma cells, we determined the effects of Aurora A silencing on glioma cells proliferation and cell cycle distribution. Our data showed that Aurora A silencing significantly suppressed cell proliferation in comparison with the control siRNA or non-treatment (parental) group both in U251 (Fig. 2A) and U87 cells (Fig. 2B).

Aurora A plays an important regulatory role for the bipolar spindle formation. Thus, it is crucial for precise chromosome segregation [27, 28]. We supposed that the proliferation inhibition of the glioma cells by RNAi-mediated Aurora A gene silencing was caused by disruption of cell cycle transition. To explore this possibility, the DNA content of cell populations reflecting the cell cycle distribution after Aurora A knockdown was assayed. A significant reduction in the $S$ phase cells after Aurora A knockdown than that of the other two groups was found in the U251 (Fig. 3A) but not in the U87 cells (Fig. 3B). This also applies to the percentage of $\mathrm{G} 2 / \mathrm{M}$ cells which were significantly varied in the U251, but not in U87 cells. The data indicated that the RNAi-mediated silencing of Aurora A not only inhibited glioma cells proliferation but also affected cell cycle distribution.

\section{Effect of Aurora A knockdown on glioma cells apoptosis}

To further confirm our findings, Annexin V/FITC and Hoechst 33258 staining assay were performed to examine the effect of RNAi-mediated Aurora A silencing on glioma cells apoptosis. Apoptosis was determined using an Annexin V/FITC kit according to the manufacturer's protocol. Aurora A siRNA significantly increased U251 cell apoptosis, neither control siRNA nor untreated group (parental, Fig. 4A). The rate of cell apoptosis in Aurora A siRNA accounted for $\sim 15 \%$, almost 3 -fold than that of the other two groups (Fig. 4B), indicating the remarkably increased cell apoptosis by RNAi.

To clarify the changes of apoptotic cell morphology after Aurora A knockdown, Hoechst 33258 staining was performed to observe nuclear changes and apoptotic body formation. Similar result that much more apoptotic body was observed after Aurora A siRNA treatment than that of the other two groups in U251 cells (Fig. 4C). Also, the numbers of the apoptotic cells, as shown in Fig. 4D, quantitated the results of nuclear staining from different treatment groups. 
(A)

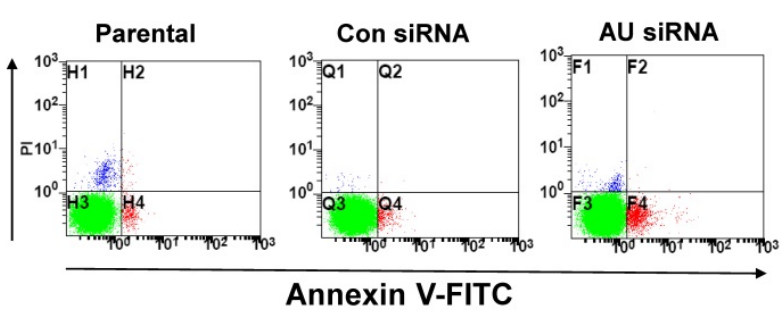

(B)

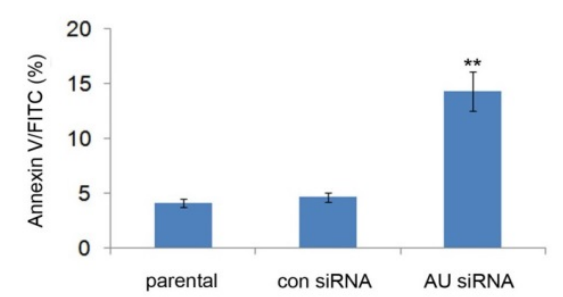

(C)

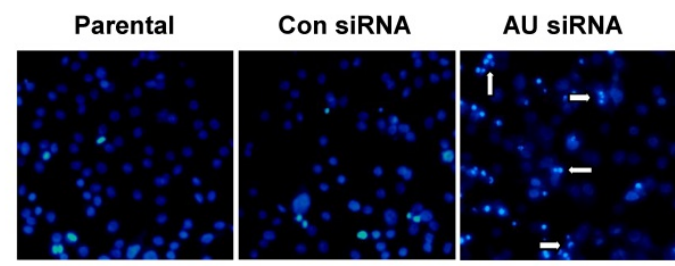

(D)

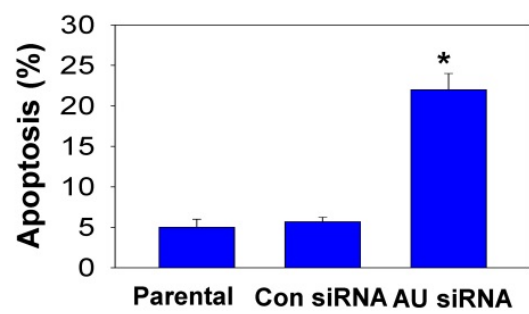

Figure 4. Effect of Aurora A silencing with siRNA on U251 cell apoptosis. U251 cells were transiently transfected with Aurora A siRNA, control siRNA or no-treated (parental). After $72 \mathrm{~h}$, cell apoptosis was assayed by Annexin V/FITC double staining and by nuclear morphology changes examination with Hoechst 33258 staining, as described in the materials and methods section. (A) The representative images of U251 cell apoptosis from different treatment groups were shown. (B) The apoptosis was summarized as the percentage of apoptotic cell numbers to total cell numbers. (C) Apoptotic cells showed typical nuclear fragmentation (white arrows) and the quantitative data showed the apoptotic ratio in various treatment groups (D). The experiments were repeated three times independently and the data were expressed as mean \pm SD from triplicate. $* \mathrm{p}<0.05,{ }^{*} \mathrm{p}<0.01$, AU indicates Aurora A, con siRNA indicates control siRNA.

To illustrate the proliferation- or apoptosisrelated molecules involved in RNAi-mediated Aurora A gene silencing leading to cell proliferation inhibition and cell apoptosis promotion, we analyzed the expression levels of cell cycle-related gene Cyclin D1, anti-apoptotic factor Bcl-2 and pro- apoptotic factor caspase-3 with western blot. As shown in Fig. 5, the Cyclin D1 and Bcl-2 protein levels were decreased, but the caspase-3 expression level was obviously increased in Aurora A siRNA treatment group compared with the other two groups.

\section{Knockdown of Aurora A suppresses tumor growth in vivo}

To explore the RNAi-mediated Aurora A silencing in glioma cells would affect the tumor growth in vivo, aliquots of $5 \times 10^{6}$ U251 cells were inoculated into the immunodeficient female nude mice and the tumor growth was monitored. No signs of possible toxicity or death were observed during the study period of time.

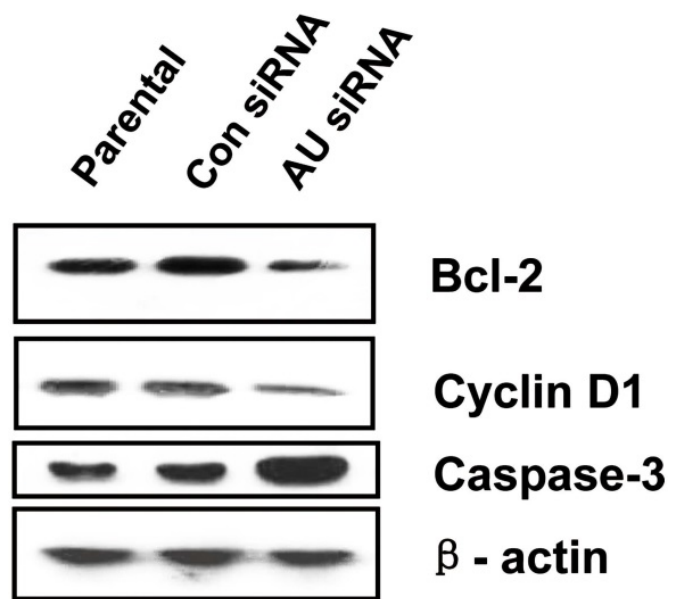

Figure 5. Effects of Aurora A silencing with siRNA on the expression of Bcl-2, Cyclin DI and Caspase-3 in U251 cells. Total proteins from Parental, control siRNA and Aurora A siRNA treatment groups were extracted and analyzed by Western blot. $\beta$-actin was used as loading control. The experiments were repeated three times independently. Aurora A knockdown in $\mathrm{U} 251$ cells resulted in decreased expression of $\mathrm{Bcl}-2$ and $\mathrm{Cyclin} \mathrm{DI}$, while increased expression of caspase-3 compared with the other two groups. $\mathrm{AU}$ indicates Aurora $\mathrm{A}$; con siRNA indicates control siRNA.

(A)

(B)
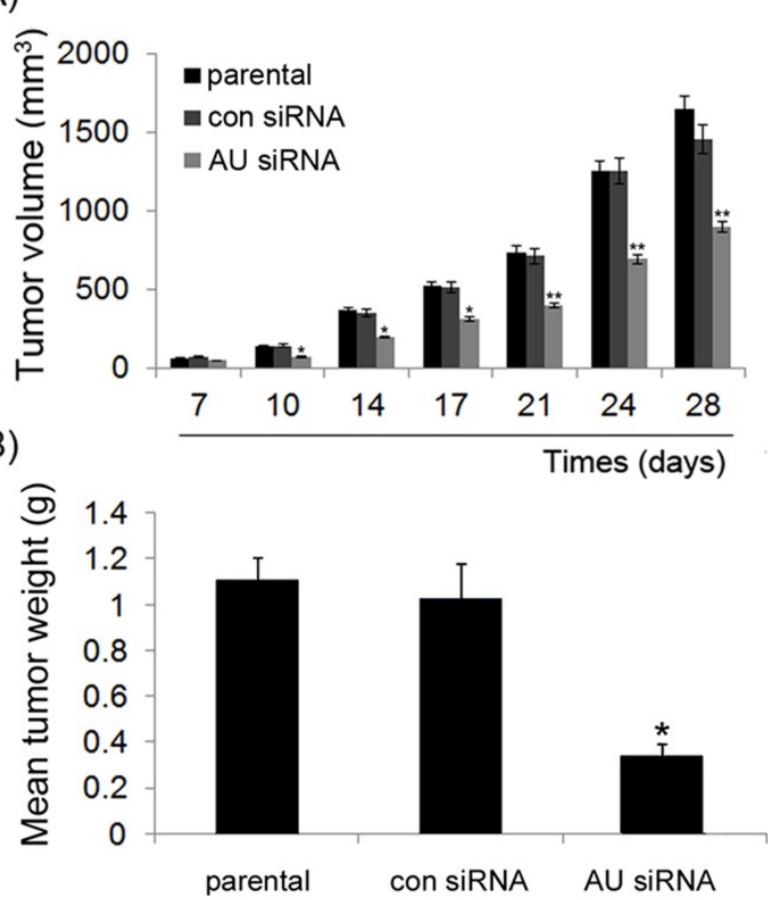

Figure 6. Effect of Aurora A silencing with siRNA on xenograft tumor growth in vivo. Intra-tumor injection of PBS (parental), Aurora A siRNA/DOPC (AU siRNA), or control siRNA/DOPC (con siRNA), respectively was performed. siRNA treatment caused a significant decrease in tumor volume $(A)$ and tumor weight $(B)$ as compared to control siRNA or PBS treatment group. Each bar represents the mean \pm SD. $n=6$, and $* p<0.05$, ** $p<0.01, A U$ indicates Aurora $A$, con siRNA indicates control siRNA. DOPC, purchased from Roche, was used for siRNA in vivo delivery. In brief, DOPC and siRNA oligos were mixed in the presence of excess tertiary butanol at a ratio of $1: 10(\mathrm{w} / \mathrm{w})$, Tween 20 was added to the mixture in a ratio of 1:19 (Tween 20:siRNA/DOPC), then the siRNA oligos were incorporated into DOPC, as described in previous study $[25,26]$. 
As illustrated in Fig. 6A, during the course of treatment, all xenograft tumor volumes increased, but significant lower growth rates appeared in the tumors that were treated with Aurora A siRNA compared to those treated with control siRNA or non-treated (parental) group at the end of the experiment. Also, at the end of the experiment, the mean tumor weight also decreased treated with Aurora A siRNA as compared to those of two other groups (Fig. 6B). Our data indicated that RNAi-mediated Aurora A gene silencing played a strong anti-tumor effect in vivo, consistent with the results of the cell proliferation in vitro, as shown in Fig. 2A, B.

\section{Discussion}

Aurora Kinases plays an important role in maintaining appropriate chromosome segregation and have been related with various of cancers. Particularly, high expression level of Aurora A has been abnormally found in a large number of cancers including breast, ovarian, and liver cancer as well as glioma when compared to their respective normal tissues [29, 30]. Previous studies have shown that Aurora A might act as a potential target for anticancer treatment in various types of cancers [17, 23, 30, 31]. Whereas, the role of Aurora A in glioma cell growth has not yet been understood. Aurora kinase inhibitors have been developed for more than one decade. The recent research data indicated that aurora kinase inhibitors promote apoptosis via mitochondrial pathway $[20,32,33]$. However, specificity, safety, and efficacy currently remain the most important challenges in the use of Aurora A inhibitors for cancer treatment $[34,35]$. Furthermore, the ATP-competitive property of the Aurora A small molecule inhibitors also reduces their specificity due to their off-target effects on other kinases. Meanwhile, more and more studies have shown that specific inhibition of Aurora A by RNA interference can effectively inhibit tumor growth [36-38]. Therefore, the development of selective siRNA is a prospective strategy for targeting Aurora A mRNA specifically expressed in glioblastoma, and RNAi is becoming a routine application for cancer treatment in vivo. In the present study, we employed the RNAi technology to knockdown the expression of Aurora A and analyze its phenotype, to explore the possibility of Aurora A as a therapeutic target. We demonstrated that knockdown of Aurora A using siRNA treatment strategy in glioma cells suppressed cell growth in vivo and in vitro.

Moreover, we showed that the knockdown of Aurora A significantly enhanced glioma cells apoptosis. The previous publications showed that the inhibition of aurora kinase could induce cell apoptosis, which is dependent on the polyploidization of cells $[39,40]$. In our work, we found that inhibition of Aurora A induced the strong accumulation in G2/M phase. Our findings are in good agreement with the previous studies by Hata et al. [23] that RNAi targeting Aurora kinase suppressed pancreatic cancer cells growth and induced cells to accumulate in G2/M phase. In addition, El-Sheikh et al. [29] suggested that RNAi-mediated knockdown of Aurora kinase A can arrest the cell cycle and reduce the viability of medulloblastoma cells. All of these observations, together with our results indicate that Aurora $\mathrm{A}$ is an attractive candidate target for cancer treatment.

It is worth noting that, in accordance with the results obtained in vitro, inhibition of Aurora A obviously decreased tumor growth in vivo than that in the control groups (Fig. 6). This result suggests that the silence of Aurora A gene could significantly inhibit the tumor cell growth in vivo, and also suggests that the knockdown strategy will be applicable in prohibiting the cancer's progression in vivo.

Studying molecular alterations is important in cancer research [41, 42]. As is well known, the oncoprotein Bcl-2 functions to inhibit or delay the induction of apoptosis in numbers of systems. Cyclin D1, one of cell cycle regulatory proteins, plays a key role in determining cell cycle transition from G1 to S phase. Whereas, caspase exists in the form of zymogens in healthy cells, which can be activated by interacting with specific adaptor proteins to promote conformational changes and autocatalytic processes, or proteolysis by already active caspase [43-47]. In our present study, we observed that the expression of Bcl-2 and Cyclin D1 were reduced by RNAi targeting against Aurora A in the U251 cells, on the contrary, the expression level of caspase-3 was upregulated obviously compared with the control groups (Fig. 5). As we all know, Bcl-2 and its homologues cause cell survival by blocking the activation of caspase [48]. Therefore, our results suggest that the cell proliferation inhibition by Aurora A knockdown was produced by modulating cell anti-apoptosis associated genes. This is also in accordance with the recent study indicating that Aurora A down-regulation resulted in increased caspase activity and enhanced cell apoptosis [30, 41]. Our work supported the claim that Aurora A targeted therapy provides an opportunity for treatment of glioma in the future, especially in high-risk patients. However, the molecular mechanism of Aurora A siRNA inducing apoptosis remains to be further elucidated.

Our study demonstrated that knockdown of Aurora A expression by siRNA strongly suppressed 
glioma cells growth in vivo and cell proliferation in vitro, as well as induced glioma cells apoptosis, indicating that Aurora A could be considered as a candidate target in gene therapy. However, the precise regulation mechanisms of Aurora A in glioblastoma remain poorly clear. Therefore, we will use new high-throughput "Omics" technologies such as genomics and epigenomics to study effect of Aurora A in glioblastoma with further analyses. In the future, we intend to analyze the interaction between transcription factors and chromosomes by $\mathrm{HiC}$ and ATAC-seq methods and find Aurora A and transcription factors related to key regions of chromosomes, and then further confirm the regulatory relationship between transcription factors-Aurora A-target genes through co-IP technology. Consequently, establishment of these regulatory relationships may open new perspectives to optimize therapies targeting epigenetic mechanisms of Aurora A in glioblastoma. With the continuous deepening of Aurora A kinase family research, the effects of Aurora $\mathrm{A}$ on mitosis and human malignancies will further clear, making it becomes a most promising tumor therapeutic targets.

\section{Conclusions}

Aurora A can be used as a candidate targeting gene and inhibition of Aurora $\mathrm{A}$ is a potentially promising therapy for glioblastoma.

\section{Supplementary Material}

Supplementary figures and tables. http://www.jcancer.org/v12p3024s1.pdf

\section{Acknowledgements}

This work was supported by the Bureau of Luoyang City Science and Technology, 2017 Medical and Health Program (1725001A-8 to Ge Zhang), and was supported by grants from the National Natural Science Foundation of China (81300207 to Haibo Wang).

\section{Author contributions}

Ge Zhang and Guanghui Ren: conceptualization, methodology, formal analysis, software; Xin Zhao and Haibo Wang: resources; Ge Zhang: investigation, data curation, writing- original draft preparation; Haibo Wang: visualization; Haibo Wang: supervision; Haibo Wang: validation; Haibo Wang: writingreviewing and editing; Haibo Wang: project administration; Ge Zhang and Haibo Wang: funding acquisition.

\section{Competing Interests}

The authors have declared that no competing interest exists.

\section{References}

1. Enloe BM, Jay DG. Inhibition of Necl-5 (CD155/PVR) reduces glioblastoma dispersal and decreases MMP-2 expression and activity. J Neurooncol. 2011; 102: 225-35.

2. Furnari FB, Fenton T, Bachoo RM, Mukasa A, Stommel JM, Stegh A, et al. Malignant astrocytic glioma: genetics, biology, and paths to treatment. Genes Dev. 2007; 21: 2683-710.

3. Jadus MR, Chen Y, Boldaji MT, Delgado C, Sanchez R, Douglass T, et al. Human U251MG glioma cells expressing the membrane form of macrophage colony-stimulating factor (mM-CSF) are killed by human monocytes in vitro and are rejected within immunodeficient mice via paraptosis that is associated with increased expression of three different heat shock proteins. Cancer Gene Ther. 2003; 10: 411-20.

4. Cui G, Li Z, Shao B, Zhao L, Zhou Y, Lu T, et al. Clinical and biological significance of nemo-like kinase expression in glioma. J Clin Neurosci. 2011; 18: $271-5$.

5. Keen N, Taylor S. Aurora-kinase inhibitors as anticancer agents. Nat Rev Cancer. 2004; 4: 927-36.

6. Giet R, Petretti C, Prigent C. Aurora kinases, aneuploidy and cancer, a coincidence or a real link? Trends Cell Biol. 2005; 15: 241-50.

7. Marumoto T, Zhang D, Saya H. Aurora-A - a guardian of poles. Nat Rev Cancer. 2005; 5: 42-50.

8. Liu Q, Ruderman JV. Aurora A, mitotic entry, and spindle bipolarity. Proc Natl Acad Sci U S A. 2006; 103: 5811-6.

9. Landen CN, Jr., Lin YG, Immaneni A, Deavers MT, Merritt WM, Spannuth WA, et al. Overexpression of the centrosomal protein Aurora-A kinase is associated with poor prognosis in epithelial ovarian cancer patients. Clin Cancer Res. 2007; 13: 4098-104.

10. Reiter R, Gais P, Jutting U, Steuer-Vogt MK, Pickhard A, Bink K, et al. Aurora kinase A messenger RNA overexpression is correlated with tumor progression and shortened survival in head and neck squamous cell carcinoma. Clin Cancer Res. 2006; 12: 5136-41.

11. Jeng YM, Peng SY, Lin CY, Hsu HC. Overexpression and amplification of Aurora-A in hepatocellular carcinoma. Clin Cancer Res. 2004; 10: 2065-71.

12. Yang SB, Zhou XB, Zhu HX, Quan LP, Bai JF, He J, et al. Amplification and overexpression of Aurora-A in esophageal squamous cell carcinoma. Oncology reports. 2007; 17: 1083-8.

13. Dar AA, Zaika A, Piazuelo MB, Correa P, Koyama T, Belkhiri A, et al. Frequent overexpression of Aurora Kinase A in upper gastrointestinal adenocarcinomas correlates with potent antiapoptotic functions. Cancer. 2008; 112: 1688-98.

14. Barton VN, Foreman NK, Donson AM, Birks DK, Handler MH, Vibhakar R. Aurora kinase A as a rational target for therapy in glioblastoma. Journal of neurosurgery. 2010; 6: 98-105.

15. Samaras V, Stamatelli A, Samaras E, Arnaoutoglou C, Arnaoutoglou M, Stergiou I, et al. Comparative immunohistochemical analysis of aurora-A and aurora-B expression in human glioblastomas. Associations with proliferative activity and clinicopathological features. Pathology, research and practice. 2009; 205: 765-73.

16. Li J, Anderson MG, Tucker LA, Shen $\mathrm{Y}$, Glaser KB, Shah OJ. Inhibition of Aurora B kinase sensitizes a subset of human glioma cells to TRAIL concomitant with induction of TRAIL-R2. Cell death and differentiation. 2009; 16: 498-511.

17. Manfredi MG, Ecsedy JA, Meetze KA, Balani SK, Burenkova O, Chen W, et al. Antitumor activity of MLN8054, an orally active small-molecule inhibitor of Aurora A kinase. P Natl Acad Sci USA. 2007; 104: 4106-11.

18. Gontarewicz A, Balabanov S, Keller G, Colombo R, Graziano A, Pesenti E, et al. Simultaneous targeting of Aurora kinases and Bcr-Abl kinase by the small molecule inhibitor PHA-739358 is effective against imatinib-resistant BCR-ABL mutations including T315I. Blood. 2008; 111: 4355-64.

19. Arbitrario JP, Belmont BJ, Evanchik MJ, Flanagan WM, Fucini RV, Hansen SK, et al. SNS-314, a pan-Aurora kinase inhibitor, shows potent anti-tumor activity and dosing flexibility in vivo. Cancer chemotherapy and pharmacology. 2010; 65: 707-17.

20. Harrington EA, Bebbington D, Moore J, Rasmussen RK, Ajose-Adeogun AO, Nakayama T, et al. VX-680, a potent and selective small-molecule inhibitor of the Aurora kinases, suppresses tumor growth in vivo. Nat Med. 2004; 10: 262-7.

21. Aihara A, Tanaka S, Yasen M, Matsumura S, Mitsunori Y, Murakata A, et al. The selective Aurora B kinase inhibitor AZD1152 as a novel treatment for hepatocellular carcinoma. J Hepatol. 2010; 52: 63-71.

22. Ochi T, Fujiwara H, Suemori K, Azuma T, Yakushijin $Y$, Hato T, et al. Aurora-A kinase: a novel target of cellular immunotherapy for leukemia. Blood. 2009; 113: 66-74.

23. Hata T, Furukawa T, Sunamura M, Egawa S, Motoi F, Ohmura N, et al. RNA interference targeting aurora kinase a suppresses tumor growth and enhances the taxane chemosensitivity in human pancreatic cancer cells. Cancer Res. 2005; 65: 2899-905.

24. Chen SW, Sun YL, Zeng ZF, Tao EX. Rifampicin inhibits apoptosis in rotenone-induced differentiated PC12 cells by ameliorating mitochondrial oxidative stress. Neural Regeneration Research. 2010; 5: 251-6.

25. Landen CN, Jr., Chavez-Reyes A, Bucana C, Schmandt R, Deavers MT, Lopez-Berestein $\mathrm{G}$, et al. Therapeutic EphA2 gene targeting in vivo using 
neutral liposomal small interfering RNA delivery. Cancer Res. 2005; 65: $6910-8$.

26. Halder J, Kamat AA, Landen CN, Jr., Han LY, Lutgendorf SK, Lin YG, et al. Focal adhesion kinase targeting using in vivo short interfering RNA delivery in neutral liposomes for ovarian carcinoma therapy. Clin Cancer Res. 2006; 12: 4916-24.

27. Hirota T, Kunitoku N, Sasayama T, Marumoto T, Zhang D, Nitta M, et al. Aurora-A and an interacting activator, the LIM protein Ajuba, are required for mitotic commitment in human cells. Cell. 2003; 114: 585-98.

28. Marumoto T, Honda S, Hara T, Nitta M, Hirota T, Kohmura E, et al. Aurora-A kinase maintains the fidelity of early and late mitotic events in HeLa cells. J Biol Chem. 2003; 278: 51786-95.

29. El-Sheikh A, Fan R, Birks D, Donson A, Foreman NK, Vibhakar R. Inhibition of Aurora Kinase A enhances chemosensitivity of medulloblastoma cell lines. Pediatr Blood Cancer. 2010; 55: 35-41.

30. Ulisse S, Arlot-Bonnemains Y, Baldini E, Morrone S, Carocci S, Di Luigi L, et al. Inhibition of the aurora kinases suppresses in vitro NT2-D1 cell growth and tumorigenicity. J Endocrinol. 2010; 204: 135-42.

31. Carmena M, Earnshaw WC. The cellular geography of aurora kinases. Nat Rev Mol Cell Bio. 2003; 4: 842-54.

32. Dar AA, Belkhiri A, Ecsedy J, Zaika A, El-Rifai W. Aurora kinase A inhibition leads to p73-dependent apoptosis in p53-deficient cancer cells. Cancer Res. 2008; 68: 8998-9004.

33. Gizatullin F, Yao Y, Kung V, Harding MW, Loda M, Shapiro GI. The Aurora kinase inhibitor VX-680 induces endoreduplication and apoptosis preferentially in cells with compromised p53-dependent postmitotic checkpoint function. Cancer Res. 2006; 66: 7668-77.

34. Borisa AC, Bhatt HG. A comprehensive review on Aurora kinase: Small molecule inhibitors and clinical trial studies. Eur J Med Chem. 2017; 140: 1-19.

35. Durlacher CT, Li ZL, Chen XW, He ZX, Zhou SF. An update on the pharmacokinetics and pharmacodynamics of alisertib, a selective Aurora kinase A inhibitor. Clin Exp Pharmacol Physiol. 2016; 43: 585-601.

36. Lai CH, Chen RY, Hsieh HP, Tsai SJ, Chang KC, Yen CJ, et al. A selective Aurora-A 5'-UTR siRNA inhibits tumor growth and metastasis. Cancer Lett. 2020; 472: 97-107.

37. Wang Z, Ma Z, Cao J. Effects of Repeated Aurora-A siRNA Transfection on Cilia Generation and Proliferation of SK-MES-1 or A549 Cells. Cancer Biother Radiopharm. 2018; 33: 110-7.

38. Zhong N, Shi S, Wang H, Wu G, Wang Y, Ma Q, et al. Silencing Aurora-A with siRNA inhibits cell proliferation in human lung adenocarcinoma cells. Int $J$ Oncol. 2016; 49: 1028-38.

39. Cheong JW, Jung HI, Eom JI, Kim SJ, Jeung HK, Min YH. Aurora-A kinase inhibition enhances the cytosine arabinoside-induced cell death in leukemia cells through apoptosis and mitotic catastrophe. Cancer Lett. 2010; 297: 171-81.

40. Dreier MR, Grabovich AZ, Katusin JD, Taylor WR. Short and long-term tumor cell responses to Aurora kinase inhibitors. Exp Cell Res. 2009; 315: 1085-99.

41. Baba Y, Nosho K, Shima K, Irahara N, Kure S, Toyoda S, et al. Aurora-A expression is independently associated with chromosomal instability in colorectal cancer. Neoplasia. 2009; 11: 418-25.

42. Wang SC, Liu H, Ren LF, Pan YF, Zhang YD. Inhibiting colorectal carcinoma growth and metastasis by blocking the expression of VEGF using RNA interference. Neoplasia. 2008; 10: 399-407.

43. Coultas L, Strasser A. The role of the Bcl-2 protein family in cancer. Semin Cancer Biol. 2003; 13: 115-23.

44. Kaabinejadian S, Fouladdel S, Ramezani M, Azizi E. Molecular analysis of $\mathrm{Bcl}-2$ and cyclin D1 expression in differentially expressing estrogen receptor breast cancer MCF7, T47D and MDA-MB-468 cell lines treated with adriamycin. Daru. 2008; 16: 182-8.

45. Stacey DW. Cyclin D1 serves as a cell cycle regulatory switch in actively proliferating cells. Curr Opin Cell Biol. 2003; 15: 158-63.

46. Thomadaki H, Scorilas A. BCL2 family of apoptosis-related genes: functions and clinical implications in cancer. Crit Rev Clin Lab Sci. 2006: 43:1-67.

47. Thornberry NA, Lazebnik Y. Caspases: enemies within. Science. 1998; 281: $1312-6$.

48. Adams JM, Cory S. The Bcl-2 protein family: arbiters of cell survival. Science. 1998; $281: 1322-6$. 